\title{
De la lecture au dialogue : García Lorca dans Un habit de Iumière
}

CARMEN MATA BARREIRO

UNIVERSITÉ AUTONOME DE MADRID

Résumé. Cet article étudie l'exploration de l'univers anthropologique et esthétique de la culture espagnole dans le roman Un habit de lumière d'Anne Hébert, et la façon dont la lecture de l'œuvre du poète et dramaturge Federico García Lorca, qui faisait partie de sa bibliothèque, enrichit cette approche. La première partie analyse la théâtralité dans ce roman et le dialogue qu'Anne Hébert établit avec certaines pièces de Lorca, dont Le public. Une deuxième partie étudie le passage du réel au mythe à l'aide d'une approche mythocritique. Une attention particulière est accordée aux symboles et mythes associés à la corrida.

Mots-clés : Espagne, García Lorca, Mythes, Symboles, La corrida, Le sacré, La mort, Théâtralité.

Dans la nécrologie concernant Anne Hébert que Gérard Meudal a écrite dans le journal Le Monde, intitulée « [L]a plus parisienne des auteures québécoises » (Meudal, 2000 : 12), il soulignait que son dernier roman, Un habit de lumière, paru en 1999, avait été présenté dans ce même journal par Viviane Forrester comme « I'une [des] [...] meilleures réussites [...] d'un des meilleurs auteurs de langue française ». (Forrester, $1999: 5$ )

Mais I'analyse de l'ensemble des articles qui traduisent la réception ${ }^{1}$ de ce roman dans les périodiques et dans des revues littéraires nous fait constater un certain dépaysement ou une certaine insatisfaction chez les critiques. Ainsi, Gilles Marcotte, tout en disant qu'« on y retrouve assurément les grands thèmes d'une œuvre toujours intensément fidèle à elle-même » (Marcotte, 1999 : 67), qualifie le roman $\mathrm{d}^{\prime}$ « étrange ». Maurice Émond commence son compte rendu dans Québec français par les paroles « C'est un bien étrange roman ». (Émond, $2000: 17$ ) Francine Bor-

1. Je tiens à remercier le service de presse des Éditions du Seuil de m'avoir fourni le dossier de presse concernant ce roman. 
deleau, dans Spirale, écrit : « [S]'il est rempli d'ironies cruelles et de violences crues, Un habit de lumière n'en demeure pas moins un roman en mineur, plus ludique qu'intense ». (Bordeleau, $2000: 10$ )

Ce qui « dépayse » dans ce roman, ce qu'on y perçoit comme étrange ou étranger, ce n'est pas la «passion, [la] fureur, [la] sensualité [ou la] violence » (Lachance, 1999 : D20), « [I] es personnages excessifs » (Chartrand, 1999 : D3), ou « sa poésie » (Péan : 1999), mais des éléments nouveaux faisant partie de la culture espagnole qui émergent dès le paratexte. En effet, le titre Un habit de lumière, qui évoque le costume, brodé de fils brillants, du torero qui a reçu l'alternative, en ayant participé à une cérémonie où l'on accorde au jeune novillero le droit d'alterner dans les courses avec les toreros, nous introduit dans l'univers de la corrida, univers qui a toujours été mis en relief dans les représentations de l'Espagne et sur lequel se sont focalisés les voyageurs canadiens-français du $19^{\mathrm{e}}$ siècle tels qu'Adolphe-Basile Routhier, qui ont adhéré à une lecture romantique de l'Espagne dans leurs récits de voyage.

Il nous semblerait inimaginable qu'Anne Hébert cultive, dans un roman, une image exotique et stéréotypée de l'Espagne, comme semble suggérer un article d'un journal de Genève, Le Courrier, qui présente Un habit de lumière comme « un roman à l'espagnole, violent, coloré, dont même le titre qui évoque la corrida joue le jeu d'un exotisme bourré de clichés. » (EWI, 1999)

Nous nous trouvons donc, en tant que lecteurs, face au problème de la lecture d'une culture. Nous, lecteurs/trices de la fin du $20^{\mathrm{e}}$ siècle et du début du $21^{\mathrm{e}}$, avons souvent de la difficulté à disséquer des couches ou strates d'une culture en évolution qui ont été banalisées et folklorisées par des hétéro-représentations mais aussi par des auto-représentations.

Anne Hébert avait déjà évoqué « Le peuple des corridas » dans le poème «Trois fois passera », qui fait partie du recueil Poèmes pour la main gauche, paru en 1997. La danse et la course des taureaux, le feu, la vie et la mort s'y engagent dans un dialogue qui atteint un degré d'intensité poétique et dramatique qui constituera l'une des caractéristiques du roman Un habit de lumière : 
La danseuse a franchi les cercles de feu

Tous les cerceaux de flamme tendus dans l'arène

Le peuple des corridas retient son souffle

Couronnée de durs reflets de forge

La voici qui rebondit pour la troisième fois

Sur la piste glacée où fument ses chaussons

Son cœur fruste en cette aventure

$Y$ passa comme une toute petite allumette.

(Poèmes pour la main gauche, $1997: 29$ )

Dans l'exploration de l'univers anthropologique et esthétique de la culture espagnole, et, plus particulièrement, andalouse, que mène Anne Hébert dans Un habit de lumière, elle a un médiateur, un « passeur » exceptionnel, l'écrivain Federico García Lorca, dont elle détenait plusieurs ouvrages dans sa bibliothèque personnelle ${ }^{2}$. En effet, sur six livres ayant trait à I'Espagne, il y a deux volumes de l'œuvre de García Lorca traduite en français, comme poète et comme dramaturge, à savoir Le romancero gitan : poème du chant profond (édition de 1995) et un volume de théâtre contenant les pièces Noces de sang, Yerma, Doña Rosita, Petit théâtre, Le jeu de Don Cristobal, Lorsque cinq ans auront passé, Le public et La maison de Bernarda (édition de 1953 contenant une date écrite par Anne Hébert : «mars 1955 »). Aborder l'étude d'Un habit de lumière en tenant compte de la lecture que l'écrivaine fait de I'œuvre de Lorca, telle qu'on peut la présumer, s'avère nécessaire en vue d'observer le parcours d'exploration d'Hébert et de repérer les points de convergence entre les deux auteurs. Dans cette approche participent la littérature comparée et les « études transculturelles » que Janet M. Paterson et Lori Saint-Martin, directrices de Voix et images. Anne Hébert en revue (2006), avaient suggérées dans leur texte de présentation (Paterson et Saint-Martin, $2006: 6$ ).

2. Je remercie Mme Christiane Bisson, responsable du Centre Anne-Hébert (Université de Sherbrooke) d'avoir répondu - avec générosité et rapidité - à mes questions sur la présence de l'œuvre de Federico García Lorca dans la bibliothèque personnelle d'Anne Hébert, et de m'avoir fourni de l'information sur tous les livres de cette bibliothèque ayant trait à I'Espagne. 


\section{Un habit de lumière : théâtralité et tragédie}

Un habit de lumière présente des allures de pièce de théâtre et se rapproche de la conception que García Lorca avait du théâtre : «Le théâtre est la poésie qui se lève du livre et devient humaine. Et en devenant humaine, elle parle et crie, pleure et se désespère. $\gg^{3}$ (García Lorca, 2004b : 83)

En ce qui concerne sa structure, le roman se divise en deux «parties». La «Première partie », dans laquelle le personnage de Miguel Almevida est un enfant - entre « [s]ept ans » et « dix ans »-, se conclut sur un énoncé elliptique : « Fin de l'enfance obscure, rue Cochin. » (Un habit de lumière, 1999 : 57) La «Deuxième partie »commence lorsque Miguel « vient tout juste d'avoir quinze ans. » $\left(H L^{4}: 64\right)$

Les personnages du roman, qui sont les membres d'une famille espagnole ayant émigré à Paris - le père, Pedro Almevida, la mère, Rose-Alba, leur fils, Miguel -, ainsi que leur voisine Mme Guillou et le « danseur étoile » du Paradis perdu, Jean-Ephrem de la Tour, s'expriment dans des monologues qui sont introduits par leurs noms. Ils se décrivent parfois eux-mêmes, et il leur arrive de parler d'eux-mêmes à la troisième personne. Anne Hébert considère «qu'il y a là un jeu de distanciation avec soi-même qui est intéressant. » (Chartrand, 1999 : D3)

La théâtralité se manifeste aussi dans la conception de l'espace. La famille Almevida habite une loge d'un immeuble où Rose-Alba se montre aux passants dès I'incipit : « C'est moi qu'on voit par la fenêtre grande ouverte de la loge. » (HL: 9) L'autre espace important, particulièrement dans la Deuxième partie, est le music-hall le Paradis perdu, sa scène, ses coulisses, sa salle, son promenoir.

C'est dans le Paradis perdu que nous retrouvons des traits communs avec un drame de García Lorca caractérisé par la liberté dans tous les domaines, sexuel, politique et théâtral - donc non présentable à l'époque de sa création - , Le public [El público, 1930], conçu lors d'un voyage de García Lorca à New York et à Cuba, en 1929-1930, et où il rend hommage à un genre de la dramaturgie classique en Espagne, l'auto sacramental. Dans El público, un metteur en scène homosexuel, dans un rêve ou en proie au délire, ose monter une version de Roméo et Juliette où les acteurs protagonistes sont un homme de 30 ans et un garçon de 15 ans. Ayant constaté l'apologie de

3. « El teatro es la poesía que se levanta del libro y se hace humana. Y al hacerse, habla y grita, llora y se desespera. » Federico García Lorca, «El teatro es la poesía que se hace humana », 7 avril 1936, dans Federico García Lorca, Teatro completo IV, 2004 : 83. Nous traduisons.

4. HL pour Un habit de lumière, 1999. 
I'homosexualité, le public qui assiste à la pièce se révolte et assassine les acteurs. Le metteur en scène rencontre la mort, déguisée en prestidigitateur, et, à la fin, il meurt. Il y a des personnages allégoriques et des symboles, et, au centre du drame, un acte sacrificiel d'origine christique : un Nu rouge portant une couronne d'épines bleues et entouré des deux Larrons.

Dans le Paradis perdu du roman d'Anne Hébert, Jean-Ephrem de la Tour, qui se présente comme «Grand de taille, petit de cœur, noir de peau, blanc de sourire, cheveux verts et bleus, [...]. Danseur au Paradis perdu [...] [qui] règne sur un peuple de danseurs et d'acrobates » $(H L: 61)$, séduit Miguel Almevida, qui « vient tout juste d'avoir quinze ans. » (HL:64) Miguel Almevida déclarera après à Jean-Ephrem : « J'aurais tant voulu être une fille et me marier avec toi. » (HL: 134) Avant son spectacle de danse, Jean-Ephrem de la Tour évoque également un acte sacrificiel, un sacrifice lié au martyre ; une isotopie fait apparaître une succession d'images « sanguinolentes » allant de la mort du taureau au martyre de Jean-Baptiste :

Ce soir la corrida sera terrible, je le sens. On me coupera peut-être les oreilles et la queue comme dans ton pays. On te fera sans doute les honneurs des oreilles et de la queue. On te les apportera sur un plat d'argent. [...] Toute la vie, toute la mort sur un plat d'argent comme la tête sanguinolente du Baptiste. (HL:68)

Un autre martyre à racine christique, à savoir une crucifixion, apparaît aussi dans le discours de Jean-Ephrem lorsqu'il décrit un portrait qu'on a fait de lui : « C'est moi, là, plumes déployées, cloué au mur par les ailes, comme une chouette sur une porte de grange » (HL: 87), crucifixion qui est aussi décrite par la mère de Miguel, Rose-Alba Almevida : «J'aime plus que jamais qu'on crucifie par les ailes Jean-Ephrem de la Tour avant de le lâcher sur la scène comme un grand papillon noir, toutes ailes battantes. » (HL: 111)

Nous repérons des emprunts intertextuels au Nouveau Testament dans Un habit de lumière et dans la pièce El público. Dans cette dernière, le personnage appelé «Desnudo » $(\mathrm{Nu})$, couronné d'épines bleues, prononce les paroles que, d'après les Évangiles, le Christ a proférées lorsqu'il était cloué sur la croix avant les trois heures de ténèbres : «Père, pardonne-leur, car ils ne savent pas ce qu'ils font. » (García Lorca, 2004a : 105), et après les trois heures de ténèbres : «Père, je remets mon esprit entre tes mains. » (García Lorca, 2004a : 106) 
En ce qui concerne la «transtextualité » (Genette, $1982: 7)$ dans Un habit de lumière, l'opération de transformation que subit le récit biblique en tant qu'hypotexte est plus explicite, car elle intervient sur la langue. Ainsi, Jean-Ephrem de la Tour, s'adressant au jeune Miguel Almevida, lui lance : «Petite Bête, tu es Petite Bête et, sur cette Petite Bête, je construirai mon bonheur. » (HL : 108) Quant à elle, Rose-Alba Almevida, dans un monologue intérieur, dit : « C'est mon fils bien-aimé en qui j'ai mis toutes mes complaisances qui me pousse à ces extrémités de songeries. » (HL: 110) Ici, I'hypertextualité aboutit à la parodie.

\section{Du réel aux espaces du mythe}

De même que dans des pièces de théâtre de García Lorca telles que Noces de sang ou Yerma, nous remarquons, dans Un habit de lumière, le passage du réel aux espaces du mythe, l'évolution du réalisme initial (à savoir un roman qui exprime les horizons d'attente d'une famille d'immigrants espagnols à Paris, leur processus d'acculturation, leurs rêves, dont certains très terre-à-terre - « Je dessine le plan de ma maison future. Ras le bol de la loge minuscule, des wc dans la cour, de l'eau sur le palier », [HL:14] -, et la nostalgie du pays d'origine) au symbolique.

Une approche mythocritique du roman d'Anne Hébert et de l'œuvre de García Lorca nous permet de repérer l'archétype de la métamorphose (Sirois, 1999 : 36) et, plus particulièrement, de celle qui concerne le règne animal. Parmi les métaphores animales qui émergent dans Un habit de lumière, nous signalerons le symbolisme du cheval et celui du taureau.

Le cheval apparaît dans Un habit de lumière (comme dans d'autres romans d'Anne Hébert) comme symbole de force et de vitalité (Sirois, 1999 : 39) et est lié à la femme. Ainsi, dès l'incipit, Rose-Alba Almevida, en décrivant son corps, fait allusion à sa « croupe rebondie ». (HL : 9) Dans la pièce de García Lorca El público, trois chevaux blancs demandent à Julieta de se déshabiller et de laisser voir sa « grupa » (croupe). (Garcia Lorca, 2004a : 94) Mais le cheval peut être également le symbole ou le messager de la mort, et dans I'œuvre de García Lorca (voir Noces de sang) et dans Un habit de lumière ${ }^{5}$. Jean-Ephrem de la Tour, dont le départ de Paris va pousser Miguel Almevida au suicide, lui demande de le percevoir comme un « cheval noir »: «Caresse mon cœur comme tu caresserais le poitrail d'un cheval noir pour le rassurer avant une course. Quelques minutes encore et je serai en piste, tout piaffant et

5. Le cheval noir apparaissait déjà comme messager de la mort dans la nouvelle « Le torrent », qu'Anne Hébert écrivit en 1944-1945. 
mort de peur. »(HL: 67-68)

Jean-Ephrem de la Tour se métamorphose aussi en taureau : « Ce soir la corrida sera terrible, je le sens. On me coupera peut-être les oreilles et la queue comme dans ton pays. On te fera sans doute les honneurs des oreilles et de la queue. » (HL:68) Mais c'est surtout le père de Miguel, Pedro Almevida, dont la hantise du déshonneur constitue la préoccupation principale, qui se métamorphose, à plusieurs reprises, en taureau, et apparaît comme symbole de violence, de « fureur. » (HL: 41) Pedro Almevida hérite de la représentation traditionnelle de I'homme espagnol, que nous retrouvons déjà en France, au $18^{\mathrm{e}}$ siècle, dans le personnage de Don Alvar du livret de Louis Fuzelier de I'opéra-ballet de Rameau, Les Indes galantes (présenté pour la première fois à Paris en 1735), chez qui l'amour comporte le désir de possession exclusive de la femme aimée, comme le souligne un autre personnage, Damon :

Les époux les plus soupçonneux

Du Tage habitent les rives,

Là, mille beautés plaintives

Reçoivent de I'hymen des fers et non des nœuds ;

Vous ne voyez jamais autour de ces captives

Voltiger les Ris et les Jeux. (Rameau, $1991: 100$ )

Cette représentation de I'homme espagnol rend Pedro Almevida gardien de I'honneur familial (« L'honneur de l'Espagne dans la ville étrangère, c'est moi, Pedro Almevida »[HL:21]), honneur qui est lié à la fidélité de la femme mariée. Chez Pedro, comme chez d'autres personnages d'hommes mariés des pièces de théâtre de García Lorca telles que Yerma, la hantise du déshonneur aboutit à la violence et entraîne la menace de mort : «Un jour, je la tuerai. » $(H L: 53)$

Lorsque Pedro découvre un manteau de fourrure chez lui, offert par un amant de Rose-Alba, la violence éclate, et il essaye d'étrangler sa femme. Leur fils, Miguel, décrit la scène en empruntant des images et des sensations au combat de la corrida :

Il y a quelque chose de terrible qui s'est passé dans la tête de mon père, quelque chose comme le chiffon rouge du taureau, agité à même la cervelle étroite de mon père, tandis que des banderilles fines étaient plantées dans son cœur, et mon père a été tout près du meurtre de ma mère, le souffle court du meurtre a été contre le visage furibond de mon père. ( $H L: 95)$

Miguel a recours de nouveau à une image de corrida associée à la violence déchaînée par la jalousie de son père, qui n'a pas pu supporter que sa femme sorte dans 
la rue habillée d'une « minijupe » qui dénude « ses gros genoux » et « ses grosses cuisses » $(H L: 22)$, et qui l'oblige à rentrer à la maison : « Ils se sont disputés. Ils se sont insultés. Ils se sont battus. Rugissant comme des taureaux dans l'arène. Se roulant par terre d'un bout à l'autre de la minuscule cuisine. Soufflant leurs derniers râlements. Au bord de la syncope ou de la crampe mortelle. » (HL:23)

Cette conception de I'honneur du mari et du père ( « Je suis le Père, le Mari, le Pater Familias » $[H L: 36])$ comporte aussi la représentation de la virilité du fils, la volonté de faire en sorte que le profil et l'identité de l'enfant se coulent dans le moule d'une virilité solide et manifeste : « [U]n fils dur et viril, enfin sorti de l'école abêtissante, [...] nerfs d'acier et bras de fer. » (HL: 71) Sa violence face à tout indice d'ambiguïté chez son fils est exprimée aussi avec des tropes où il est comparé au taureau :

Il I'a fait exprès. Il l'a prise par les pieds et jetée par terre dans la cuisine. Ma pauvre poupée en cent morceaux. Fracassée. Une poupée d'autrefois très cassante et superbe. Il était tout rouge comme un torchon qu'on agite devant le taureau pour l'exciter. Lui-même taureau, mon père, respirant fort dans sa fureur. Il répétait comme un toqué.

- Pas de ça, fiston! Pas de ça ! Jamais ! (HL: 41)

Il est là qui me surveille à travers des bouffées bleues. Ce n'est pas le moment de le contredire. Il a l'air de méditer une sortie de taureau dans l'arène. Je marche sur la pointe des pieds en passant devant lui. Surtout il ne faut pas qu'il sache que je saute à la corde à la récré, comme une fille, malgré sa défense. ( $H L: 56)$

Symboles et mythes : la corrida, le sacré et la mort

Le cheval et le taureau convergent dans la corrida, qu'Anne Hébert présente comme un espace de fête et de mort, de séduction et de peur. La coexistence de la fête et de la mort, de la lumière et de l'ombre, dans la représentation de la corrida, et l'évolution, inéluctable, vers la mort, apparaissent particulièrement, d'une façon symétrique, au début de la première et de la deuxième parties du roman. En effet, au début du roman, Miguel Almevida joue avec des craies de couleur et essaie de métamorphoser « le gris de l'ennui. Rouge, vert, bleu, jaune, blanc, violet. Je pose la couleur sur l'ennui étale du trottoir » $(H L: 14)$, tandis que sa mère rêve et l'imagine en « vrai petit torero »: 
Mon fils unique, Miguel, est avec moi, dans le même éclat insoutenable. Un vrai petit torero en habit de lumière. Qu'il soit nu ou habillé, mon fils brille et moi, sa mère, je brille avec lui. Olé ! olé ! j'entends les cris de la foule en délire. C'est mon fils qu'on acclame. Je respire la poussière brûlante de l'arène. Le galop furibond de la Bête en agonie me passe sur la face. L'odeur du sang et de la mort me poursuit jusque dans la ville étrangère où je suis concierge, 102, rue Cochin, dans le Ve, Paris, France. (HL : 10)

Ce rêve de Rose-Alba Almevida nous fait penser au quatrième acte de I'opéra Carmen de Bizet, qui met en scène le jour d'un combat de taureaux, et fait apparaître une musique fortement colorée, très ensoleillée, où le chœur crie « Victoire ! ». Ces fanfares joyeuses mettront mieux en valeur, par contraste, la fin si sombre, un esprit que Nietzsche définissait ainsi : «C'est la fièvre de la passion proche de la mort ». (Jean de Solliers, 1980 : 84) Comme le personnage de Carmen dans cet opéra, Miguel accepte son destin dans la deuxième partie. Il assume la nouvelle signification de «la fête », et le nouveau décor, «la nuit » et «le noir des rues », et il s'engage dans une nouvelle période déterminée par le rapport $\mathrm{d}^{\prime} \ll$ obéissance » à «l'Ange des Ténèbres » :

J'ai tant attendu cette nuit pleine de périls, sans savoir que je l'attendais, frileusement, dans I'usure des jours enfantins, [...]. Voici que la fête pour laquelle je suis fait de toute éternité bat en sourdine tel un cœur frémissant, derrière les murs du Paradis perdu. Me suis faufilé dans le noir des rues, pour la première fois, seul et endimanché, dans la nuit. [...] [I]l est I'Ange des Ténèbres, né pour se perdre et me perdre avec lui. [...] Il me dit va et je vais. Il me dit viens et je viens. De mon obéissance dépend mon bonheur. (HL: 65-66)

L'alliage de séduction et de peur est particulièrement intense dans la relation entre Jean-Ephrem de la Tour et Miguel Almevida, dont la première rencontre a eu lieu « vers les cinq heures du soir » $(H L: 62)$, heure qui est mise en relief dans un poème que García Lorca a consacré à un torero, passionné de littérature et de danse andalouse, Ignacio Sánchez Mejías, à savoir le « Chant funèbre pour Ignacio Sanchez Mejias » («Llanto por Ignacio Sánchez Mejías » [1934]) : 


\section{LA BLESSURE ET LA MORT}

À cinq heures du soir.

Il était juste cinq heures du soir.

Un enfant apporta le drap blanc

à cinq heures du soir.

Un couffin de chaux préparé

à cinq heures du soir.

Et tout n'était que mort et rien que mort

à cinq heures du soir.

$[\ldots]$

Et le taureau avec son cœur debout !

à cinq heures du soir. (García Lorca, 1981 : 75-76)

Miguel Almevida est « [f]igé de peur » lorsque Jean-Ephrem de la Tour s'adresse à lui en l'appelant « petite bête. » (HL:62) Miguel vit cette relation comme une initiation, comme l'accès à une nouvelle étape de sa vie, comme une profonde métamorphose : «Le monde s'ouvre devant moi. Encore un peu et je connaîtrai les secrets de la terre. [...] Je ne serai plus jamais le même après cela, vêtu d'enfance comme d'un vêtement étriqué. » (HL: 66, 69)

Jean-Ephrem jouit de cette peur qu'il provoque chez l'adolescent et qui va devenir le lien entre eux :

Déjà lié à lui, l'enfant effrayé, par la peur visible sur toute sa petite personne recroquevillée dans un escalier, rue Saint-Victor, vers les cinq heures du soir. Tel est son destin sans doute, être effrayé par moi. Tel est le mien sans doute, mettre le comble à une terreur déjà ancienne, comme originelle, en lui. ( $H L: 62)$

Jean-Ephrem lui explique que, pour lui, le mot « bête » est « sacré » et que « les dieux seuls ont le droit de s'appeler ainsi. » (HL:67) Il lui demande de l'appeler « Belle Bête » et introduit entre eux une double dimension dans leur rapport, « animal et sacré » : «Et tout sera égal entre nous, animal et sacré. » (HL:67)

Ce rapport où interviennent la séduction et la peur, l'animal et le sacré inhérent à la corrida, fait aussi penser à La minotauromachie ${ }^{6}$ (1935) de Pablo Picasso, gravure 
qui illustre le combat entre les forces du mal, les ténèbres de la nuit, incarnées par le Minotaure, qui s'avance, menaçant, vers une petite fille, et celles du bien, de la lumière, de l'innocence, représentées par la petite fille. Picasso y mêle un personnage de la mythologie grecque, le Minotaure, fils d'une reine de Crète, Pasiphaé, et d'un taureau sorti de la mer, et qui est donc mi-homme, mi-taureau et mi-dieu, avec sa mythologie propre à lui, celle de son pays, la corrida, ce combat tragique et mortel entre I'homme et I'animal.

Un habit de lumière et le Romancero gitan : le mythe, les archanges « éphèbes », le chromatisme

Plusieurs éléments rapprochent le roman Un habit de lumière et le recueil Romancero gitan de García Lorca, que l'écrivain présentait en 1935, lors d'une conférence-récital donnée à la Résidence des étudiants de Barcelone, comme «le poème de l'Andalousie »: « [J]e l'appelle gitan parce que le gitan est ce qu'il y a de plus élevé, de plus profond, de plus aristocratique dans mon pays, de plus représentatif de sa manière et ce qui conserve la braise, le sang et l'alphabet de la vérité andalouse et universelle. » (García Lorca, 1981 : 62-63) Et il ajoutait : « [L]e livre est un retable de I'Andalousie avec des gitans, des chevaux, des archanges, des planètes, avec sa brise juive, avec sa brise romaine, avec des rivières, avec des crimes [...]. Un livre où est à peine exprimée l'Andalousie que I'on voit et où frémit celle que I'on ne voit pas. » (García Lorca, 1981 : 63)

Tout le Romancero gitan est consacré à la mythologie gitane, et García Lorca y juxtapose l'actualisation de mythes et de types de la mythologie gitane traditionnelle et la réinterprétation et l'intégration, dans la mythologie gitane, des martyres de la période paléochrétienne (voir «Martyre de sainte Eulalie ») ou des récits bibliques tels que I'histoire de Thamar et Amnon. Il y fait converger des types mythiques masculins et féminins qui évoluent dans des paysages andalous aux maisons blanchies à la chaux (cf. «Mort d'amour ») et aux champs d'oliviers (voir «Précieuse et le vent »); des anges («Rixe »); et des archanges (« Saint Michel», « Saint Raphaël », « Saint Gabriel »); la déesse lune dotée d'un pouvoir maléfique ( «Romance de la lune »); et les traits christiques de la mort de Juan Antonio de Montilla dans le poème «Rixe » : 
Juan Antonio de Montilla

dévale en mourant la pente,

le corps étoilé d'iris,

une grenade à la tempe,

et sur une croix de flamme

prend la route de la mort. (García Lorca, 1981 : 16)

Tout le Romancero gitan, la violence, la mort, l'érotisme tragique, font partie d'un seul élément, personnage ou thème, qui s'infiltre et s'empare des êtres humains et du paysage. Il s'agit de la « peine andalouse », sentiment tragique de la vie, andalou et panthéiste, lutte de l'intelligence amoureuse avec le mystère qui l'entoure et qu'elle ne peut saisir7. D'après Allen Josephs et Juan Caballero, toute l'esthétique de Lorca est synthétisée dans la peine andalouse, et c'est cette esthétique qu'il personnifie et rend mythique dans le Romancero gitan.

Miguel et les archanges « éphèbes »

Dans le recueil de García Lorca, trois poèmes sont consacrés aux archanges, « San Miguel » («Saint Michel »), «San Rafael » («Saint Raphaël ») et « San Gabriel » («Saint Gabriel »), qui, comme le rappelait le poète (1935), « expriment les trois grandes Andalousies »: Grenade, Cordoue et Séville. San Miguel est présenté, dès le début de son poème, comme un éphèbe, un «Éphèbe aux trois mille nuits », qui, «Dans la loge de sa tour / [...] / montre ses superbes cuisses / moulées par les lampadaires. » (García Lorca, 1981 : 28) San Gabriel est décrit comme « Un bel enfant de jonc, / larges épaules, taille mince, / le teint de pomme nocturne, / grands yeux, bouche douce-amère » et aux « souliers de cuir verni ». (García Lorca, 1981 : 32) San Rafael apparaît comme « L'Archange à demi arabe / sous ses paillettes obscures ». (García Lorca, 1981 : 31)

Miguel Almevida est décrit par son père comme «ce petit qui hésite encore entre fille et garçon » qui, lorsqu'il sort avec lui, porte « une chemise blanche » et des « souliers pointus et brillants». (HL:21) Lorsque Miguel devient adolescent, JeanEphrem de la Tour souligne certains traits physiques chez lui : «Fin à l'extrême. Avec de grands yeux vides » (HL:83-84), « magnifiques les jambes, des vraies jambes de fille ». (HL : 106) 
Le chromatisme : dimensions symbolique et structurante

L'importance du chromatisme est équivalente dans le Romancero gitan et dans Un habit de lumière. Ainsi, nous retrouvons les « anges noirs » du poème «Rixe » et les «noirs chevaux» de la «Romance de la Garde civile espagnole», associés à la mort et au deuil, de même que l'« Ange des Ténèbres » (HL:66) ou I'« Ange noir » ( $H L: 110)$ dans Un habit de lumière. Mais ce qui s'avère plus significatif est le chromatisme comme un axe structurant dans les deux ouvrages. Dans le Romancero gi$\tan ^{8}$, dans la série de romances autour de quatre types mythiques féminins (IV-VII : «Romance somnambule », «La nonne gitane », «La femme infidèle » et « Romance de la peine noire »), la couleur verte traverse la « Romance somnambule » tandis que c'est la couleur noire qui teinte la dernière romance en mettant en scène la douleur ou la « peine noire » de Soledad Montoya. Un autre exemple est le rapport entre la dernière romance de la première partie, «Romance de la Garde civile espagnole », et la première romance de la deuxième partie ou série de "Trois romances historiques », à savoir le « Martyre de sainte Eulalie ». Dans la « Romance de la Garde civile espagnole », les deux couleurs qui l'emportent sont le noir associé à la mort, aux cendres, au deuil, et le rouge du sang et des flammes destructrices. Dans la première « romance historique » se succèdent la couleur rouge du sang de la martyre, le contraste entre le noir et le blanc de l'encre, du charbon et de la neige, et la couleur blanche de la fin du poème accompagnant la vision mystique de la martyre.

Dans Un habit de lumière, la lumière, l'ombre et les couleurs signalent le rythme, les tensions et les luttes chez les personnages, ainsi que la progression vers le dénouement tragique. Dans la première partie, où la lumière du jour l'emporte, la recherche des couleurs vives, voire voyantes, que mènent les membres de la famille Almevida vise à métamorphoser le paysage urbain de Paris, qu'ils perçoivent comme une « ville grise » $(H L: 21)$, de même que leur vie dans la «loge minuscule» $(H L: 14)$ qu'ils se représentent comme un «[t]rou à rats ». (HL: 34)

Dès l'incipit, Rose-Alba, se laissant aller à une rêverie, « où tout est [...] splendeur » (HL : 11), imagine une scène resplendissante : «Mon fils unique, Miguel, est avec moi, dans le même éclat insoutenable. Un vrai petit torero en habit de lumière. Qu'il soit nu ou habillé, mon fils brille et moi, sa mère, je brille avec lui. » (HL:10) Cette rêverie est nourrie par « les craies de couleur » $(H L: 11)$ que son fils Miguel utilise,

8. Voir Christian de Paepe, «El Romancero gitano. Estudio de Christian de Paepe », dans Federico García Lorca, Romancero gitano, 2006 : 51-52. 
« $[r]$ ouge, vert, bleu, jaune, blanc, violet » $(H L: 14)$, pour triompher du « gris de l'ennui », de I'« ennui étale du trottoir. » $(H L: 14)$ Mais la fragilité du rêve de la mère et le caractère éphémère de l'action de l'enfant se manifestent. La pensée de la mort s'insinue dans le discours de Rose-Alba et l'obsède : « L'odeur du sang et de la mort me poursuit » $(H L: 10)$, tandis que Miguel voit que « [l] a craie crisse entre [s]es doigts, s'effrite et s'écrase » $(H L: 14)$ et que les passants effacent le dessin coloré.

Le soleil est évoqué dans les comparaisons associées aux couleurs voyantes de I'univers de Rose-Alba, à ses robes « d'or comme un soleil abrégé » (HL:22), et à la couleur que celle-ci choisit pour se teindre les cheveux, «blond vénitien » (HL:48), en vue de se transformer en « [t] oute une splendeur dorée sortie de l'ombre comme un soleil éclatant, trop longtemps tenu captif. » (HL: 48) Chez Pedro Almevida, c'est la blancheur de sa chemise et de celle de son fils, à côté de lui, qui lui fait penser à « [I]'honneur de l'Espagne dans la ville étrangère » et à «la Fiesta » $(H L: 21)$, tandis que surgit le souvenir du parfum « des brassées de jasmin. » (HL : 20) Et lorsqu'il rêve de retourner en Espagne, il imagine une maison «blanchie à la chaux. [...] [avec] [u]n petit champ de vigne à côté ». (HL: 37)

Dans la deuxième partie, la nuit et l'obscurité l'emportent sur la lumière. Miguel Almevida apprend à apprivoiser la nuit de Paris, qu'il avait sentie comme hostile et louche lors de sa fugue provoquée par la violence de son père. La nuit devient un espace initiatique pour lui, prêt à suivre Jean-Ephrem de la Tour et à se soumettre à lui, «l'Ange des Ténèbres ». (HL:66) Miguel est fasciné par «le soleil noir des fêtes nocturnes » $(H L: 69)$ et se sent « libre de courir vers d'étranges félicités » $(H L: 72)$, « libre comme l'air noir des nuits ». (HL : 81) Le Paradis perdu apparaît comme un lieu où rivalisent les lumières et les ombres (« son ombre sur le mur est celle d'un géant », $H L: 66)$, un lieu de clair-obscur, peuplé par « les garçons et les filles à peine pubères, transfigurés sous les feux de la rampe »(HL:63), que Miguel voit passer avant le spectacle, « en pleine mutation d'anges ou de démons. » ( $H L: 67)$

Lorsque Jean-Ephrem de la Tour le quitte, Miguel se sent abandonné, « [c]hassé de partout. » (HL : 136) Perçu par Jean-Ephrem de la Tour comme une « petite épave destinée au feu du ciel » (HL: 117), Miguel se laisse attirer par la Seine et, captivé par un mirage, retrouve «des images à moitié rêvées dans l'eau frissonnante » ( $H L$ : 136), en y rejoignant les paysages andalous pleins de couleurs évoqués dans son enfance : «L'Espagne de mes parents est là, avec ses maisons blanches, ses oliviers d'argent, ses vignes vertes ». (HL : 136-137) À I'intérieur de cette illusion visuelle, Miguel croit percevoir « [q]uelqu'un de sacré [...] [qui] [lui] prépare en secret 
[...] un habit de lumière pour quand [il sera] arrivé parmi les morts. » ( $H L: 137)$

Du « Torrent » à Un habit de lumière : la « douloureuse Espagne » de García Lorca

Le discours de Miguel annonçant le dénouement tragique du roman Un habit de lumière, son suicide, confirmé au moyen d'une focalisation interne par l'entremise du personnage de la voisine Mme Guillou («Le fils s'est noyé dans la Seine, la mère hurle si fort qu'on l'entend jusque dans la rue »[HL: 137]), nous fait penser à une autre scène, mirage ou hallucination, qui clôt la nouvelle « Le torrent », texte majeur d'Anne Hébert publié dans le recueil éponyme en 1950. Une comparaison entre les deux écrits s'avère pertinente en vue d'analyser la présence de certaines constantes et de certaines variantes et de pouvoir ainsi mieux percevoir l'influence de l'œuvre de García Lorca dans Un habit de lumière.

La nouvelle «Le torrent » relate la vie de François Perrault, dévoilée à travers un monologue intérieur qui traduit un travail d'anamnèse d'une « conscience souffrante qui tente de faire du sens du non-sens. » (Picard-Drillien, 2006 : 95) Les derniers mots du monologue intérieur révèlent la force d'attraction du torrent, qui fait partie de la topographie de François et qui semble l'amener inéluctablement à la mort : « Je suis fatigué de regarder l'eau et d'y cueillir des images fantastiques. Je me penche tant que je peux. Je suis dans l'embrun. Mes lèvres goûtent l'eau fade. [...] Je veux voir le gouffre, le plus près possible. Je veux me perdre en mon aventure, ma seule et épouvantable richesse. » (《Le torrent », $2011: 44$ )

Dans « Le torrent», nous sommes face au discours d'un adulte, François, pour qui le torrent constitue une force pulsionnelle, qui fait partie d'un paysage auquel il se soumet tout en le faisant sien. Soumission et voie d'affranchissement y interagissent : «Il n'y a de vivant que le paysage autour de moi. [...] Je suis identifié au paysage. » $\left(T^{9}: 25\right)$ Et cet adulte croit percevoir dans l'eau noire du torrent des images, telles que «la tête d'Amica [...] arrachée » $(T: 44)$, qui sont des échantillons d'un délire paranoïaque qui est la dernière expression d'un désir destructeur et de la perversion.

$\mathrm{Si}$, chez François, la haine, le secret effroyable lié à la mort de sa mère et la perversion entraînent la mort ou le meurtre de l'enfant qu'il a été et l'impossibilité pour l'adulte de survivre à ce meurtre, chez Miguel, l'enfant qu'il a été n'est pas mort et 
dans son suicide ; il rejoint des paysages andalous qui le réconfortent et des images de corrida auxquelles une dimension sacrée est associée.

Une autre différence concerne la relation entre la mère et le fils. Dans « Le torrent », la violence, l'incommunicabilité et la volonté despotique de domination chez Claudine déterminent « une impuissance, une stérilité » $(T: 18)$ chez François, de même que l'angoisse et la haine. Chez Rose-Alba, la complicité et l'amour pour son fils coexistent avec la rivalité ou la jalousie. Lors des fugues de Miguel, Rose-Alba montre son désespoir et compare sa souffrance avec celle $d^{\prime}$ « une martyre d'autrefois dans les couvents d'Espagne. » (HL: 44) Lorsqu'elle apprend la mort de son fils, elle « hurle » $(H L: 137)$ en agissant comme le personnage de la concierge de la pièce de théâtre de García Lorca, Lorsque cinq ans auront passé, qui pleure lorsqu'on va enterrer son enfant, et dont on entend la voix criant « Mon fils ! Mon fils ! (Garcia Lorca, 2004a : 138, 176)

Dans Un habit de lumière, de même que dans d'autres écrits hébertiens, nous retrouvons des tensions, jamais résolues, entre la soumission et l'affranchissement, le bien et le mal, le jour et la nuit, la lumière et l'ombre, la vie et la mort. Anne Hébert s'y rapproche de «I'esprit caché de la douloureuse Espagne » (García Lorca, 2009 : 11), de l'Espagne comme « pays de mort. [...] pays ouvert à la mort » (García Lorca, 2009 : 37) que García Lorca évoque dans Jeu et théorie du duende. Elle y montre qu'elle a appréhendé « la liturgie de la corrida, authentique drame religieux, où, de même qu'à la messe, on adore et on sacrifie un dieu. » (García Lorca, $2009: 51,53)$ 


\section{Bibliographie}

BERNADAC, Marie-Laure et Paule DU BOUCHET (1999) [1986], Picasso. Le sage et le fou, Paris, Gallimard, coll. « Découvertes ».

BORDELEAU, Francine (2000), «Les vertiges de la nuit : Un habit de lumière d'Anne Hébert », Spirale, $\mathrm{n}^{\circ} 172: 10$.

CHARTRAND, Robert (1999), « La distance nécessaire : L'auteur de Kamouraska raconte son septième roman », Le Devoir, 19-20 juin : D3.

ÉMOND, Maurice (2000), « Anne Hébert : Un habit de lumière », Québec français, $n^{\circ} 117: 17$.

EWI (1999), Mère et fils à la fois complices et ennemis », Le Courrier, 5 juin.

FORRESTER, Viviane (1999), «Scintillements trompeurs », Le Monde, 18 juin : 5.

GARCÍA LORCA, Federico (1981) [1961], Romancero gitan suivi de Chant funèbre pour Ignacio Sanchez Mejias, trad. André Belamich, Paris, Gallimard.

GARCÍA LORCA, Federico (1988), Primer romancero gitano, Llanto por Ignacio Sánchez Mejías, Madrid, Castalia.

GARCÍA LORCA, Federico (2004a), « El público », dans Teatro completo, II, Barcelone, Random House Mondadori, DeBolsillo : 67-118.

GARCÍA LORCA, Federico (2004b), Teatro completo, IV, Barcelone, Random House Mondadori, DeBolsillo.

GARCÍA LORCA, Federico (2006) [1977], Poema del Cante Jondo. Romancero gitano, Allen Josephs et Juan Caballero (dir.), Madrid, Cátedra

GARCÍA LORCA, Federico (2006) [1928], Romancero gitano, dans Christian de Paepe (dir.), Madrid, Espasa Calpe, coll. « Austral Poesía ».

GARCÍA LORCA, Federico (2009) [2008], Jeu et théorie du duende, trad. Line Amselem, Paris, Allia.

GENETTE, Gérard (1982), Palimpsestes : la littérature au second degré, Paris, Seuil.

HÉBERT, Anne (2011) [1963, 1950], « Le torrent » dans Le torrent, Montréal, Hurtubise.

HÉBERT, Anne (1997), Poèmes pour la main gauche, Montréal, Boréal.

HÉBERT, Anne (1999), Un habit de lumière, Paris, Seuil.

LACHANCE, Lise (1999), « Passions sans issue », Le Soleil, 10 juillet : D20.

MARCOTTE, Gilles (1999), « Où se trouve le paradis perdu ? », L'Actualité, $1^{\mathrm{er}}$ septembre : 67-68.

MATA BARREIRO, Carmen (2012), « Anne Hébert », dans Ursula Mathis-Moser et Birgit MertzBaumgartner (dir.), Passages et ancrages en France. Dictionnaire des écrivains migrants de langue française (1981-2011), Paris, Honoré Champion, coll. « Dictionnaires et Références », n 23 : 404-409.

MATA BARREIRO, Carmen (2012), Espagnes imaginaires du Québec, Québec, Presses de l'Université Laval.

MEUDAL, Gérard (2000), «Anne Hébert. La plus parisienne des auteures québécoises », Le Monde, 25 janvier : 12.

PATERSON, Janet M. et Lori SAINT-MARTIN (2006), « Présentation », dans Anne Hébert en revue, Québec/Montréal, Presses de l'Université de Québec/Voix et images : 1-7. 
PÉAN, Stanley (1999), « Quand tombent les anges », Le Couac, août.

PICARD-DRILLIEN, Anne-Marie (2006), " L'enfant du “ Torrent “ ou le sujet de l'œuvre en puissance », dans Janet Paterson et Lori Saint-Martin (dir.), Anne Hébert en revue, Québec/Montréal, Presses de l'Université de Québec/Voix et images : 93-114.

RAMEAU, Jean-Philippe (1991), Les Indes galantes (Livret de Louis Fuzelier), Arles, Harmonia mundi. SIROIS, Antoine (1999), Lecture mythocritique du roman québécois, Montréal, Triptyque.

SOLLIERS, Jean de (1980), « Commentaire littéraire et musical », L’Avant-scène, n 26 : 23-94. 